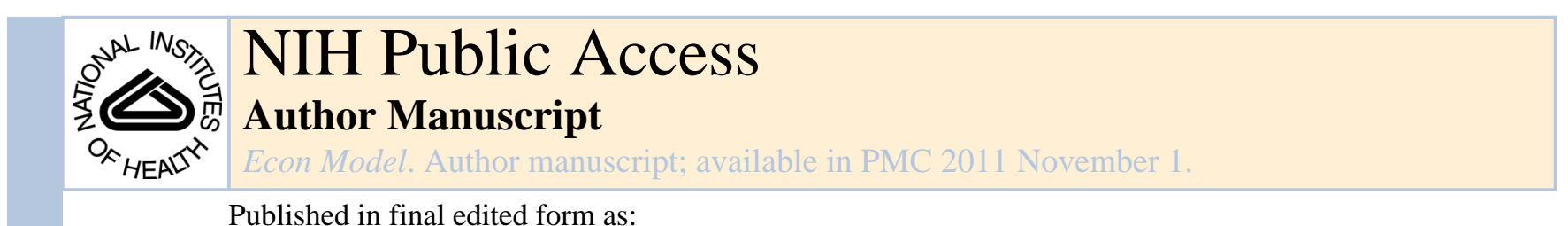

Econ Model. 2010 November 1; 27(6): 1355-1367. doi:10.1016/j.econmod.2010.07.019.

\title{
Tests of Hypotheses Arising In the Correlated Random Coefficient Model ${ }^{\star}$
}

\author{
James J. Heckman and \\ University of Chicago, University College Dublin, Cowles Foundation, Yale University, and the \\ American Bar Foundation
}

Daniel Schmierer

University of Chicago

\begin{abstract}
This paper examines the correlated random coefficient model. It extends the analysis of Swamy (1971), who pioneered the uncorrelated random coefficient model in economics. We develop the properties of the correlated random coefficient model and derive a new representation of the variance of the instrumental variable estimator for that model. We develop tests of the validity of the correlated random coefficient model against the null hypothesis of the uncorrelated random coefficient model.
\end{abstract}

\section{Keywords}

Random coefficient models; correlated random coefficient models; instrumental variables

\section{Introduction}

The correlated random coefficient model is the new centerpiece of a large literature in microeconometrics. It extends the classical uncorrelated random coefficient model of Swamy $(1971,1974)$. For person $i$, outcome $Y_{i}$ in terms of choice indicator $D_{i}$ is written as

$$
Y_{i}=\alpha_{i}+\beta_{i} D_{i}
$$

where $D_{i}=1$ if a choice is made; $D_{i}=0$ if not and both the intercept, $\alpha_{i}$, and the slope, $\beta_{i}$, vary among persons. In this expression both the $\alpha_{i}$ and $\beta_{i}$ may depend on regressors $X_{i}$ which we keep implicit.

$\beta_{i}$ is the causal effect of $D_{i}$ on $Y_{i}$ holding $\alpha_{i}$ fixed. If agents make their choices to take treatment based on components of $\beta_{i}$ that depend on variables not available to the observing

(C) 2010 Elsevier B.V. All rights reserved.

James Heckman: Department of Economics, University of Chicago, 1126 East 59th Street, Chicago, IL 60637; phone, 773-702-0634; fax, 773-702-8490; jjh@uchicago.edu. Daniel Schmierer: Department of Economics, University of Chicago, 1126 East 59th Street, Chicago, IL 60637; phone, 773-702-1787; fax, 773-702-8490; dschmier@uchicago.edu..

*The website for this paper is http://jenni.uchicago.edu/testing_random/.

Publisher's Disclaimer: This is a PDF file of an unedited manuscript that has been accepted for publication. As a service to our customers we are providing this early version of the manuscript. The manuscript will undergo copyediting, typesetting, and review of the resulting proof before it is published in its final citable form. Please note that during the production process errors may be discovered which could affect the content, and all legal disclaimers that apply to the journal pertain. 
economist, $D_{i}$ is correlated with $\beta_{i}$ even after conditioning on $X_{i}$. Most recent studies focus on estimating means or quantiles of the distribution of $\beta_{i}{ }^{1}$

The model that motivated the research of a previous generation (see, e.g., Griliches, 1977) assumes no response heterogeneity $\left(\beta_{i}=\beta\right)$ or else an uncorrelated random coefficient model as in Swamy $(1971,1974)$ or Mincer $(1974)$, so $\beta_{i}$ is independent of $D_{i}$. The correlated random coefficient model assumes that $\beta_{i}$ varies in the population and in addition that

$$
\operatorname{Cov}\left(D_{i}, \beta_{i}\right) \neq 0 \text {. }
$$

The model also accounts for selection on intercepts, i.e. selection on pretreatment unobservables:

$$
\operatorname{Cov}\left(D_{i}, \alpha_{i}\right) \neq 0
$$

When (C-1) holds, marginal returns to an activity in general differ from average returns. When assumption (C-2) holds but $D_{i}$ is independent of $\beta_{i}$, standard IV identifies the mean of $\beta_{i}$, which we denote by $\beta^{-}$. This configuration of assumptions includes the case when $\beta_{i}$ is random but independent of $D_{i}$ and the case when $\beta_{i}$ is the same for everyone. ${ }^{2,3}$

As first noted by Heckman and Robb (1985), instrumental variables (IV) applied to (1) when (C-1) holds produces an instrument-dependent parameter that, in general, is not $\beta^{-4}$ In general, different instruments identify different parameters. Under conditions specified in Yitzhaki (1989), ${ }^{5}$ Imbens and Angrist (1994), Heckman and Vytlacil (1999), and Heckman, Urzua, and Vytlacil (2006), IV estimates weighted averages of marginal effects. Heckman and Vytlacil (1999, 2001, 2005, 2007a) generalize the marginal treatment effect (MTE) introduced by Björklund and Moffitt (1987) and show that the MTE plays the role of a functional that is invariant to the choice of instrument. The MTE can be used to unify the literature on treatment effects. ${ }^{6}$

Heckman and Vytlacil $(2001,2005,2007 b)$ derive testable implications of the hypothesis that $\beta_{i}$ is statistically independent of $D_{i}$ given $X_{i}$ :

$$
H_{0}: \beta_{i} \Perp D_{i} \mid X_{i},
$$

where $A \Perp B \mid C$ means $A$ is independent of $B$ given $C$. In this paper, we examine tests of this hypothesis, drawing heavily on our previous work (Heckman, Schmierer, and Urzua, 2010). We also consider tests of the hypothesis that $E\left(\beta_{i}\right)=\beta^{-}=0$ and that the IV estimand of (1) is zero.

The paper proceeds as follows. Section 2 establishes the equivalence of the correlated random coefficient model with the Generalized Roy model. We examine two testable implications of it. One test exploits the insight that, in general, in the case when $H_{0}$ is false, different instruments identify different parameters. Section 3 presents some new results on

\footnotetext{
${ }^{1}$ Abbring and Heckman (2007) discuss methods for estimating the distribution of $\beta_{i}$.

${ }^{2}$ See Heckman and Vytlacil (1998), Heckman and Vytlacil (2007a,b). The standard "ability bias" problem (Griliches, 1977) assumes that $i=\beta$, a constant for all $i$, and that $\operatorname{Cov}\left(D_{i}, \alpha_{i}\right) \neq 0$.

${ }^{3}$ Evidence from parametric models on the empirical relevance of $(\mathrm{C}-1)$ in a variety of areas of economics is presented in Heckman (2001, Table 3).

${ }_{5}^{4}$ See the discussion of the ensuing literature in Heckman, Urzua, and Vytlacil (2006) or Heckman and Vytlacil (2007a,b).

5 Posted at website for Heckman, Urzua, and Vytlacil (2006), see http://jenni.uchicago.edu/underiv/.

${ }^{6}$ See Heckman and Vytlacil $(2005,2007 a, b)$.
} 
the sampling distribution of the instrumental variable estimator and develops tests for $\beta^{-}=0$ and for the IV estimand to be zero. Section 4 develops the tests of the correlated random coefficient model. Section 5 develops other tests of $H_{0}$ and places all tests in the conditional moment testing framework (Ai and Chen, 2003). Section 6 concludes.

\section{Equivalence with the Generalized Roy Model and Two Testable Implications of $H_{0}$}

An alternative way to represent equation (1) makes the link to economic choice theory more explicit. Individual $i$ experiences outcome $Y_{1, i}$ if $D_{i}=1$ and outcome $Y_{0, i}$ if $D_{i}=0, i=1, \ldots$, $I$. The observed outcome is $Y_{i}=D_{i} Y_{1, i}+\left(1-D_{i}\right) Y_{0, i}{ }^{7}$ Let $\mu_{j}\left(X_{i}\right)=E\left(Y_{j, i} \mid X_{i}\right), j \in\{0,1\}$. One can write the model for potential outcomes conditional on $X_{i}$ as $Y_{1, i}=\mu_{1}\left(X_{i}\right)+U_{1, i}$ and $Y_{0, i}=\mu_{0}\left(X_{i}\right)+U_{0, i}$ where $E\left(U_{j, i} \mid X_{i}\right)=0, j \in\{0,1\}$. In this notation, the observed outcome is

$$
Y_{i}=\mu_{0}\left(X_{i}\right)+\left[\mu_{1}\left(X_{i}\right)-\mu_{0}\left(X_{i}\right)+U_{1, i}-U_{0, i}\right] D_{i}+U_{0, i}
$$

This is the correlated random coefficient model of equation (1) where the baseline outcome is $\alpha_{i}=\mu_{0}\left(X_{i}\right)+U_{0, i}$ and the gain is $\beta_{i}=\mu_{1}\left(X_{i}\right)-\mu_{0}\left(X_{i}\right)+U_{1, i}-U_{0, i}$ where, for notational simplicity, we suppress the dependence of $\alpha_{i}$ and $\beta_{i}$ on $X_{i}$. To simplify the expressions, we drop the $i$ subscripts throughout the rest of the paper unless their use clarifies the discussion. We define $\alpha=\alpha^{-}+U_{\alpha}$ and $\beta=\beta^{-}+U_{\beta}$ where $E\left(U_{\alpha} \mid X\right)=0$ and $E\left(U_{\beta} \mid X\right)=0$. Table 1 shows the equivalent parameters for the two models.

Whether the null hypothesis $H_{0}$ is true or not depends on the underlying choice model. We postulate a threshold crossing model which assumes separability between observables $Z$ that affect choice and an unobservable $V: D=\mathbf{1}\left(\mu_{D}(Z)-V>0\right)$, where $1(\cdot)$ is an indicator function that takes the value 1 if its argument is true and is 0 otherwise, and $\mu_{D}$ is a deterministic function of $Z .{ }^{8} Z$ can include components of $X$. Letting $F_{V}$ be the distribution of $V$ conditional on $X$, and assuming that $Z \Perp V \mid X$, the choice probability or "propensity score" is

$$
P(z)=\operatorname{Pr}(D=1 \mid Z=z)=F_{V}\left(\mu_{D}(z)\right),
$$

where to simplify the notation, we keep the conditioning on $X$ implicit. The choice equation can be written in several alternative and equivalent ways:

$$
D=1\left(\mu_{D}(Z)-V>0\right)=1\left(F_{V}\left(\mu_{D}(Z)\right)>F_{V}(V)\right)=1\left(P(Z)>U_{D}\right)
$$

where $U_{D}=F_{V}(V)$ so $U_{D} \sim$ Uniform $[0,1]$.

We invoke the assumptions of Heckman and Vytlacil $(2005,2007 \mathrm{~b}) .{ }^{9}$ A fundamental treatment parameter introduced by Björklund and Moffitt (1987) is the marginal treatment effect (MTE). The MTE for a given value of $X=x$ is

\footnotetext{
${ }^{7}$ This is in the form of a Quandt (1958) switching regression model.

${ }^{8}$ See, e.g., Thurstone (1927) and McFadden $(1974,1981)$. We do not strictly require separability, but we do require that the choice equation has one representation in separable form. See Heckman and Vytlacil (2007b).
} 


$$
\operatorname{MTE}\left(x, u_{D}\right)=E\left(Y_{1}-Y_{0} \mid X=x, U_{D}=u_{D}\right)=E\left(\beta \mid X=x, U_{D}=u_{D}\right) .
$$

It is the mean effect of treatment when the observables $X$ are fixed at a value $x$ and the unobservable in the choice equation $U_{D}$ is fixed at a value $u_{D}$. Heckman and Vytlacil $(1999,2001,2005,2007 \mathrm{~b})$ use the MTE to develop implications of the model to test $H_{0}$.

In the general case, the conditional expectation of $Y$ given $X$ and $Z$ is

$$
\begin{aligned}
E(Y \mid X= & x, Z=z) \\
& =E(Y \mid X=x, P(Z)=p) \\
& =E(\alpha \mid X=x) \\
& +E(\beta D \mid X=x, P(Z)=p) \\
& =E(\alpha \mid X=x) \\
& +E(\beta \mid X=x, D=1) p \\
& =E(\alpha \mid X=x) \\
& +\int_{0}^{p} E\left(\beta \mid X=x, U_{D}=u_{D}\right) d u_{D},
\end{aligned}
$$

where the integrand in the final expression is the $\operatorname{MTE}\left(x, u_{D}\right) \cdot{ }^{10}$ Under $H_{0}$,

$$
E\left(\beta \mid X=x, U_{D}=u_{D}\right)=E(\beta \mid X=x),
$$

so

$$
E(Y \mid X=x, P(Z)=p)=E(\alpha \mid X=x)+E(\beta \mid X=x) p .
$$

11 Thus the function $E(Y \mid X=x, P(Z)=p)$ is linear in $p$, conditional on $X=x$, which is a testable hypothesis.

A second implication of $H_{0}$ is that any standard instrument identifies $\bar{\beta}=E(\beta) .{ }^{12}$ Thus under $H_{0}$ all valid instruments have the same estimand. Under conditions presented in this paper, comparing the estimates produced by different instruments tests the weaker hypothesis

$H_{0}^{\prime}: \operatorname{Cov}(\beta, D \mid X)=0$, which is an implication of the stronger hypothesis $H_{0}$. The analysis in this paper thus provides an alternative interpretation of standard tests of overidentification. A rejection of the null hypothesis that two instrumental variable estimands are different is

\footnotetext{
${ }^{9}$ Their conditions are:

(A-1) $\left(U_{0}, U_{1}, V\right) \Perp Z \mid X$. Alternatively, $(\alpha, \beta, V) \Perp Z \mid X$.

(A-2) The distribution of $\mu_{D}(Z)$ conditional on $X$ is nondegenerate. Thus the distribution of $P(Z)$ is nondegenerate conditional on $X$.

(A-3) The distribution of $V$ is continuous (i.e., absolutely continuous with respect to Lebesgue measure). Thus $U_{D}=F_{V}(V)$ is uniform.

(A-4) $E\left|Y_{1}\right|<\infty$, and $E\left|Y_{0}\right|<\infty$, so defining $\left.E(\beta)=\beta^{-}, \mid \beta\right\rceil<\infty$.

(A-5) $1>\operatorname{Pr}(D=1 \mid X)>0$.

Vytlacil (2002) shows that under mild regularity conditions, assumptions (A-1)-(A-5) are equivalent to the IV conditions of Imbens and Angrist (1994) used to define the local average treatment effect (LATE).

10 The first line follows from (A-1). The rest of the derivation comes from (1) and the law of iterated expectations.

${ }^{11}$ To see this, notice that $\beta \Perp D\left|X \Leftrightarrow \beta \Perp \mathbf{1}\left(P(Z)>U_{D}\right)\right| X \Leftrightarrow \beta \Perp U_{D} \mid X$ given (A-1).

${ }^{12}$ In the notation of equation (1), but dropping subscripts $i$, a standard instrument $J$ has the two properties: (i) $\operatorname{Cov}(J, D \mid X) \neq 0$ and (ii) $\operatorname{Cov}((\alpha, \beta), J \mid X)=0$. Note that $J$ is shorthand for $J(Z)$. Note further that the condition $\operatorname{Cov}(\beta, J \mid X)=0$ only emerges as an interesting condition in a random coefficient model.
} 
not necessarily a rejection of the validity of one instrument. It could be interpreted as evidence in support of a correlated random coefficient model.

\section{General Properties of the IV Estimator for the Correlated Random Coefficient Model and Tests of the Hypotheses $\beta^{-}=0$ and that the IV Estimand Is Zero}

We present a new representation of the sampling distribution of the IV estimator. We consider the problem of constructing the power of tests of several hypotheses using the sampling distribution of the IV estimator for the correlated random coefficient model.

\subsection{IV in the Correlated Random Coefficient Model}

Consider an instrument $J(Z)$. Denote $J(Z)$ by $J$ and define $\tilde{J}=J-\bar{J}$ where $J^{-}$is the sample mean of $J(Z) . E(J)$ is assumed to be finite. The IV estimator is

$$
\widehat{\beta}_{I V, J}=\frac{\sum Y_{i} \tilde{J}_{i}}{\sum \tilde{D}_{i} \tilde{J}_{i}} .
$$

Define $\operatorname{Cov}(J, D)=\omega_{J}$ and let $I$ denote the sample size. Under a weak law of large numbers, $\frac{1}{I} \sum D_{i} \tilde{J}_{i} \stackrel{p}{\rightarrow} \omega_{J}$ and $\bar{J} \stackrel{p}{\rightarrow} E(J)$. As shown in Heckman and Vytlacil $(2005,2007 \mathrm{~b})$, under the conditions (A-1)-(A-5) stated in Section 2,

$$
\widehat{\beta}_{I V, J} \stackrel{p}{\rightarrow} \beta_{I V, J}=\int_{0}^{1} E\left(\beta \mid U_{D}=u_{D}\right) h_{J}\left(u_{D}\right) d u_{D}
$$

where

$$
h_{J}\left(u_{D}\right)=\frac{E\left[(J-E(J)) \mid P(Z)>u_{D}\right] \operatorname{Pr}\left(P(Z)>u_{D}\right)}{\omega_{I}},
$$

and we keep the conditioning on $X$ implicit. Heckman and Vytlacil (2005) show that $\int_{0}^{1} h_{J}(t) d t=1$. Thus we can write

$$
\beta_{I V, J}=\bar{\beta}+\int_{0}^{1} E\left(U_{\beta} \mid U_{D}=u_{D}\right) h_{J}\left(u_{D}\right) d u_{D} .
$$

For later use we break out the component of $\beta_{I V, J}$ that depends on the instrument $J$ :

$$
\int_{0}^{1} E\left(U_{\beta} \mid U_{D}=u_{D}\right) h_{J}\left(u_{D}\right) d u_{D}=\mathrm{Y}_{J}
$$

so $\beta_{I V, J}^{-}=\beta^{-}+\mathrm{Y}_{J}$. By definition, conditional on $X, \beta^{-}$does not depend on $J$.

Under independent sampling,

$$
\sqrt{I}\left(\widehat{\beta}_{I V, J}-\beta_{I V, J}\right) \stackrel{d}{\rightarrow} N\left(0, \Omega_{J}\right)
$$


where

$\Omega_{J}=E\left[\alpha^{2}\right] \frac{\operatorname{Var}(J)}{\omega_{J}^{2}}+\int_{0}^{1}\left[2 E\left(\alpha \beta \mid U_{D}=u_{D}\right)+E\left(\beta^{2} \mid U_{D}=u_{D}\right)\right] h_{\Omega_{J}}\left(u_{D}\right) d u_{D}-\left(\int_{0}^{1} E\left(\beta \mid U_{D}=u_{D}\right) h_{J}\left(u_{D}\right) d u_{D}\right)^{2}$

and

$h_{\Omega_{J}}\left(u_{D}\right)=\frac{1}{\omega_{J}^{2}} \int_{-\infty}^{\infty}(j-E(J))^{2} \int_{u_{D}}^{1} f_{P, J}(P(z), j) d P(z) d j=\frac{E\left[(J-E(J))^{2} \mid P(Z)>u_{D}\right] \operatorname{Pr}\left(P(Z)>u_{D}\right)}{\omega_{J}^{2}}$.

13 The weight $h_{\Omega J}\left(u_{D}\right)$ does not necessarily integrate to 1 :

$$
\int_{0}^{1} h_{\Omega_{J}}(t) d t=\frac{\operatorname{Cov}\left(\tilde{J}^{2}, D\right)}{[\operatorname{Cov}(\tilde{J}, D)]^{2}} .
$$

Appendix A presents the full derivation. The weight $h_{\Omega_{J}}\left(u_{D}\right)$ plays a role in determining the variance of the IV estimator that is analogous to the role of $h_{J}\left(u_{D}\right)$ in generating the probability limit of the IV estimator. $2 E\left[\alpha \beta \mid U_{D}=u_{D}\right]+E\left[\beta^{-2} \mid U_{D}=u_{D}\right]$ plays a role in generating the variance of the IV estimator analogous to the role of the MTE in generating the probability limit of the IV estimator. We use this representation to facilitate comparison of the power of the tests under alternative data generating processes and to consider the problem of the optimal choice of instruments.

These formulae hold for general functions $J(\cdot)$ of instruments $Z$ that satisfy assumptions (A-1)-(A-5) given in Section 2. For example, suppose that $J(Z)$ has discrete support on points $j_{1}, \ldots, j_{K}$ with corresponding values of the propensity score $p_{1}, \ldots, p_{L}$ with $L$ possibly not equal to $K$. Let $p_{0}=0$. In this case, for $u_{D} \in\left(p_{l}, p_{l+1}\right)$ both $h_{J}$ and $h_{\Omega_{j}}$ are constant so we can write

$$
\Omega_{J}=E\left[\alpha^{2}\right] \frac{\operatorname{Var}(J)}{\omega_{J}^{2}}+\sum_{l=0}^{L-1} \lambda_{\Omega_{l}} \int_{p_{l}}^{p_{l+1}}\left[2 E\left(\alpha \beta \mid U_{D}=u_{D}\right)+E\left(\beta^{2} \mid U_{D}=u_{D}\right)\right] \frac{1}{p_{l+1}-p_{l}} d u_{D}-\left(\sum_{l=1}^{L-1} \lambda_{l} \int_{p_{l}}^{p_{l+1}} E\left(\beta \mid U_{D}=u_{D}\right) \frac{1}{p_{l+1}-p_{l}} d u_{D}\right)^{2}
$$

The weights $\lambda_{\Omega_{l}}$ and $\lambda_{l}$ are defined in the following way. Let $j_{i}$ be the $i$ th smallest value in the support of $J(Z)$, then

$$
\begin{aligned}
& \lambda_{\Omega_{l}}=\frac{\sum_{i=1}^{K}\left[j_{i}-E(J)\right]^{2} \sum_{l>l}^{L} f_{P, J}\left(p_{t}, j_{i}\right)}{\operatorname{Cov}(\tilde{J}(Z), D)^{2}}\left(p_{l+1}-p_{l}\right) \\
& \lambda_{l}=\frac{\sum_{i=1}^{K}\left[j_{i}-E(J)\right] \sum_{D>l}^{L} f_{P, J}\left(p_{t}, j_{i}\right)}{\operatorname{Cov}(\tilde{J}(Z), D)}\left(p_{l+1}-p_{l}\right) .
\end{aligned}
$$

${ }^{13} f_{P, J}(P(z), j)$ is the density of $P(Z)$ and $J(Z)$ evaluated at $P(Z)=P(z)$ and $J(Z)=j$. 
The special case of a binary instrument $J(Z)$ has two points of support, $j_{1}$ and $j_{2}$, corresponding to the points $p_{1}$ and $p_{2}$ in the propensity score distribution. $\operatorname{Let} \operatorname{Pr}\left(J(Z)=j_{1}\right)=$ $\operatorname{Pr}\left(P(Z)=p_{1}\right)=q$ and $\operatorname{Pr}\left(J(Z)=j_{2}\right)=\operatorname{Pr}\left(P(Z)=p_{2}\right)=1-q$. The $\lambda_{l}$ are $\lambda_{1}=1$ and $\lambda_{l}=0, l>$ $1 .{ }^{14}$ The weights for the variance simplify to

$$
\lambda_{\Omega_{0}}=\frac{\left[j_{1}-E(J)\right]^{2} q+\left[j_{2}-E(J)\right]^{2}(1-q)}{\operatorname{Cov}(\tilde{J}(Z), D)^{2}}\left(p_{1}\right) \quad \text { and } \quad \lambda_{\Omega_{1}}=\frac{\left[j_{2}-E(J)\right]^{2}(1-q)}{\operatorname{Cov}(\tilde{J}(Z), D)^{2}}\left(p_{2}-p_{1}\right) \text {, }
$$

and

$$
\lambda_{\Omega_{0}}+\lambda_{\Omega_{1}}=\frac{\left(j_{1}-E(J)\right)^{2} q p_{1}+\left(j_{2}-E(J)\right)^{2}(1-q) p_{2}}{\operatorname{Cov}(\tilde{J}, D)^{2}}=\frac{\operatorname{Cov}\left(\tilde{J}^{2}, D\right)}{\operatorname{Cov}(\tilde{J}, D)^{2}} .
$$

Formula (4) extends the representation of IV as weighted averages of slopes of the underlying function, due to Yitzhaki (1989). It allows the instrument $J(Z)$ be different from the propensity score $P(Z)$ or a monotonic function of it. It reveals that, in general, different instruments identify different parameters. Thus, in general, $\beta_{I V, J} \neq \beta_{I V, J}$, if $J$ and $J$ ' apply different weights (5) to a common MTE.

As noted by Heckman and Vytlacil (2005,2007b), while the weight in (5) integrates to 1, it is not necessarily non-negative for all values of $u_{D}$ so the interpretation of the weighted average produced by IV is obscure. Even though the MTE is positive everywhere, the IV estimate may be negative. ${ }^{15}$

Some applied economists report tests based on IV sampling distributions as if they are testing the null hypothesis that $\beta^{-}=0$. Under $H_{0}$, i.e., the absence of a correlated random coefficient model, the sampling distribution of the standard IV estimator, $\hat{\beta}_{I V, J}$, can be used to consistently test the null hypothesis that $\beta^{-}=0$. However, when $H_{0}$ is false, a test of $\beta^{-}=$ 0 based on the sampling distribution of the IV estimator is, in general, inconsistent and biased because by (6), IV does not, in general, converge to $\beta^{-}$.

Consider the following example based on the normal generalized Roy Model,

$$
\left(\begin{array}{c}
U_{1} \\
U_{0} \\
V
\end{array}\right) \sim N\left(\left(\begin{array}{l}
0 \\
0 \\
0
\end{array}\right),\left(\begin{array}{ccc}
\sigma_{1}^{2} & \sigma_{10} & \sigma_{1 V} \\
\sigma_{10} & \sigma_{0}^{2} & \sigma_{0 V} \\
\sigma_{1 V} & \sigma_{0 V} & \sigma_{V}^{2}
\end{array}\right)\right)
$$

14

$$
\lambda_{1}=\frac{\left[j_{2}-E(J)\right](1-q)}{\operatorname{Cov}(\tilde{J}(Z), D)}\left(p_{2}-p_{1}\right)=\frac{\left(j_{2}-j_{1}\right)\left(p_{2}-p_{1}\right) q(1-q)}{\operatorname{Cov}(\tilde{J}(Z), D)}=\frac{\operatorname{Cov}(\tilde{J}(Z), P(Z))}{\operatorname{Cov}(\tilde{J}(Z), D)}=1
$$

${ }^{15}$ See the examples in Heckman, Urzua, and Vytlacil (2006). 
and assume $X=1$. Recalling that $u_{D}=F_{V}(v)$, when $V$ is a normal random variable, the marginal treatment effect is

$$
\operatorname{MTE}\left(U_{D}=u_{D}\right)=\bar{\beta}+\left(\frac{\sigma_{1 V}-\sigma_{O V}}{\sigma_{V}}\right) \Phi^{-1}\left(u_{D}\right)
$$

where $\Phi^{-1}(\cdot)$ is the inverse of a standard normal CDF (hence $\Phi^{-1}\left(u_{D}\right)=v$ ). Alternatively, in terms of $v$,

$$
\operatorname{MTE}(V=v)=\bar{\beta}+\frac{\sigma_{1 V}-\sigma_{0 V}}{\sigma_{V}} v
$$

Let $\tau=\frac{\sigma_{1 V}-\sigma_{O V}}{\sigma_{V}}$. A value of $\tau \neq 0$ produces a correlated random coefficient model. For such values plim $\beta_{I V, J}^{-} \neq \beta^{-}$. The choice equation is assumed to be $D=\mathbf{1}(Z>V)$ where both $Z$ (a single instrument) and $V$ are normally distributed and $Z \Perp V$. Additionally, assume that $\sigma_{1}^{2}=\sigma_{0}^{2}=\sigma_{t}^{2}, \sigma_{10}=0.5 \times \sigma_{1} \times \sigma_{0}$ and $\sigma_{V}^{2}=1$.

Figure 1 plots the power of a Wald test of the hypothesis that $\beta^{-}=0$ based on $\hat{\beta}_{I V, J}$. We compute the power function for different values of $\beta^{-}$. Recall from (6) that this is the component of $\beta_{I V, J}^{-}$that does not depend on $J$. In Panel A, $\hat{\beta}_{I V, J}$ is a consistent estimator for $\beta^{-}$. In the other two panels it is not. Thus in the top panel of the figures, when $\tau=0$, and hence $H_{0}$ is true, the test of the hypothesis $\bar{\beta}=0$ is unbiased and consistent and the size of the test is controlled. ${ }^{16}$ As expected, smaller values of $\sigma_{V}^{2}$ produce higher power, and larger values of $\sigma_{Z}^{2}$ produce higher power. The bottom two panels plot the power of the test that $\bar{\beta}=0$ when $\tau=-1$ and $\tau=0.6$, respectively. In these two latter cases, $\operatorname{plim} \widehat{\beta}_{I V, J}=\beta_{I V, J} \neq \bar{\beta}$. Hence the tests are biased and inconsistent. The power and size of the test for the existence of an "effect" (i.e., whether $\bar{\beta}=0$ ) can be badly distorted. Thus even if $\bar{\beta}=0$, an "effect" can be detected, and if $\bar{\beta} \neq 0$, no "effect" can be detected.

\subsection{Testing Hypotheses About Instrument-Dependent Parameters}

More recently, many applied economists, following Imbens and Angrist (1994), interpret IV as a weighted average of "LATEs," or in our framework, a weighted average of MTEs, as in equation (3). It is understood that $\hat{\beta}_{I V, J}$ is not, in general, consistent for the true $\beta^{-}$. Within this framework, economists often report tests of the hypothesis that $\beta_{I V, J}=0$.

To calculate the power of such tests, consider alternative values of $\beta_{I V, J}\left(=\beta^{-}+\mathrm{Y}_{J}\right.$ from equation (6)) obtained by varying $\beta^{-}$holding $\mathrm{Y}_{J}$ fixed. Notice that unlike the analysis in the preceding section, in this section we are not testing the hypothesis that $\beta^{-}=0$. Instead we are testing the hypothesis that $\beta_{I V, J}=0$ (or some other specified value). We vary $\beta^{-}$to calculate the power of the test for alternative values of $\beta_{I V, J}$. This is a sensible way to proceed because $\beta^{-}$is instrument invariant. Investigating the power of the test in this fashion allows us to construct power functions for instrument-invariant alternatives.

Figure 2 plots the power function for the Wald test of the hypothesis $\beta_{I V, J}=0$ as a function of $\beta_{I V, J}$ holding $\mathrm{Y}_{J}$ fixed at -0.5 . Consequently, the $\beta^{-}$compatible with the null hypothesis,

\footnotetext{
${ }^{16}$ Although Figure 1 shows the power function only for one sample size, the consistency of the test is readily verified.
} 
$\beta_{0}^{-}$, is 0.5 . For the model of unobservables used in the previous subsection, keeping $\mathrm{Y}_{J}$ fixed entails, among other things, holding $\tau=\frac{\sigma_{1 V}-\sigma_{0 V}}{\sigma_{V}}$ fixed along with the weighting function $h_{J}\left(u_{D}\right)$. For a given $\tau$ and a fixed IV weighting function $h_{J}\left(u_{D}\right)$, we vary the parameters of covariance matrix (9). These parameters affect the sampling distribution of $\hat{\beta}_{I V, J}$ and hence the power of the test.

Neither the IV estimand nor the variance of the IV estimator depends on $\sigma_{10}$. Therefore, the power of the test of the null hypothesis $\beta_{I V, J}=0$ does not depend on $\sigma_{10}$. The only remaining parameters that can be changed without changing are $\mathrm{Y}_{J}$ are $\sigma_{0}^{2}, \sigma_{1}^{2}, \sigma_{1 V}$ and $\sigma_{0 V}$. To keep $\tau$ fixed, we can only vary $\sigma_{1 V}$ and $\sigma_{0 V}$ subject to a constraint that $\sigma_{1 V}-\sigma_{0 V}$ is constant. ${ }^{17}$ For $\sigma_{V}=1$, the four A panels of Figure 2 show the power of the test for different values of $\beta^{-}$ when we vary $\sigma_{1 V}$ and $\sigma_{0 V}$ such that $\sigma_{1 V}-\sigma_{0 V}=-1$. The power of the test is highest when $\sigma_{1 V}$ and $\sigma_{0 V}$ are both close to 0 (ie. straddling 0 ), and lowest when both are far from zero (either positive or negative). The panels in B vary $\beta_{I V, J}$ by varying $\beta^{-}$holding $\mathrm{Y}_{J}$ fixed and hold fixed all of the parameters of (9) except for $\sigma_{1}^{2}$, while the panels in C vary $\beta^{-}$hold fixed all of the parameters of (9) except $\sigma_{0}^{2}$. As expected, power decreases as both variances increase, in general at different rates.

There are other ways to calculate the power of the test that $\beta_{I V, J}=0$ for alternative values that are obtained by varying $\beta^{-}$keeping $\mathrm{Y}_{J}$ fixed. If the choice equation is

$$
D=1(Z \gamma>V)
$$

and $Z \sim N\left(\bar{Z}, \Sigma_{Z}\right)$ and $V \sim N\left(0, \sigma_{V}^{2}\right)$, all instruments constructed from linear or affine transformations of $Z$ have the same weight function (5) and hence have the same instrumentdependent value, $\beta_{I V, J}$. For proof of this claim, see Appendix B. ${ }^{18}$

This result implies that one can construct power functions for the hypothesis $\beta_{I V, J}=0$ for different values of $\beta_{I V, J}=\beta^{-}+\mathrm{Y}_{J}$ for alternative choices of $\Sigma_{Z}$, holding $\gamma^{\prime} \Sigma_{Z} \gamma$, the variance of the choice index, constant. The derivation in Appendix B shows that the IV estimand depends only on the distribution of the index $Z \gamma-V$. From assumption (A-1), $Z \gamma$ and $V$ are statistically independent. $\sigma_{V}^{2}$ has to be held constant to keep $\mathrm{Y}_{J}$ fixed. We keep this term fixed by varying components of $Z$ while keeping $\gamma^{\prime} Z \gamma$ fixed. An instrument with greater variance that obeys this constraint will produce greater power. Figure 3 plots power functions of the test of the hypothesis that $\beta_{I V, J}=0$ using each component of a twodimensional instrument $Z=\left(Z_{1}, Z_{2}\right)$. These plots show that for a given IV estimand $\beta_{I V, J}^{-}$, the power of the test is higher when using the instrument that accounts for more of the variance of the index $Z \gamma$. Going from top to bottom, the variance of $Z_{1}$ is increasing while the variance of $Z_{2}$ is decreasing. Accordingly, from top to bottom the power of the test $\beta_{I V, J}$ $=0$ using $Z_{1}$ as an instrument is increasing while the power of the test using $Z_{2}$ as an instrument is decreasing. Each panel shows the fraction of $\gamma^{\prime} Z \gamma$ accounted for by the variance of the instrument used to construct the power function (either $Z_{1}$ or $Z_{2}$ ). ${ }^{19}$ We now use the tools developed for IV in a correlated random coefficient model to test $H_{0}$.

\footnotetext{
17 Variations in $\sigma_{V}^{2}$ affect the denominator of the weights.

${ }^{18}$ This result is special to the case of $J(Z)$ linear or affine in $Z$ with $Z$ normally distributed, so $J(Z)$ is normally distributed and the further assumption (A-1) that $Z \Perp V$, where $V$ is normally distributed. We have not analyzed more general conditions on $Z$ and $V$ under which the invariance holds.

${ }^{19}$ Note that in a given row, the fractions do not sum to 1 because there is a covariance (of 0.1 ) between $Z_{1}$ and $Z_{2}$.
} 


\section{Testing $H_{0}$ Using Instrumental Variables}

Armed with the results of Section 3, we study how to use different IVs to test $H_{0}$. Under $H_{0}$, the probability limits of any two IV estimators are identical, because for any choice of $J$,

$$
\operatorname{plim} \widehat{\beta}_{I V, J}=\beta_{I V, J}=\int_{0}^{1} E\left(\beta \mid U_{D}=u_{D}\right) h_{J}\left(u_{D}\right) d u_{D}=\bar{\beta} \int_{0}^{1} h_{J}\left(u_{D}\right) d u_{D}=\bar{\beta} .
$$

If $H_{0}$ is false, in general any two IV estimators will differ. Excluding the case of equal IV weights for the two instruments, our IV test forms two estimators $\widehat{\beta}_{I V, 1}$ and $\widehat{\beta}_{I V, 2}$, based on $J_{1}(Z)$ and $J_{2}(Z)$ respectively, and tests the null hypothesis

$$
H_{0}^{I V}: \beta_{I V, 1}-\beta_{I V, 2}=0
$$

against the alternative hypothesis

$$
H_{A}^{I V}: \beta_{I V, 1}-\beta_{I V, 2} \neq 0
$$

This test is identical to a standard test for overidentification. However, within the context of a correlated random coefficient model, we do not interpret rejections of the null hypothesis as evidence of the violation of the assumptions required for the validity of an instrument. Rather, rejections are interpreted as evidence of selection on heterogeneous gains to treatment.

Under the null hypothesis, the Wald test statistic is asymptotically distributed as a $\chi_{1}^{2}$. Under the alternative, in the general case, the Wald statistic converges to a noncentral chi-square distribution. Let $h_{1}(\cdot)$ and $h_{2}(\cdot)$ denote the weights (akin to $h_{J}(\cdot)$ above) corresponding to $J_{1}(Z)$ and $J_{2}(Z)$, respectively. To simplify the notation, we suppress the $Z$ argument. Define $\tilde{J}_{1}=J_{1}-\bar{J}_{1}$ and $\tilde{J}_{2}=J_{2}-\bar{J}_{2}$ as the demeaned values of the instruments. Let $\tilde{\mathbf{J}}_{1}=\left(\tilde{J}_{11}, \ldots, \tilde{J}_{1 I}\right)^{\prime}$ and $\tilde{\mathbf{J}}_{2}=\left(\tilde{J}_{21}, \ldots, \tilde{J}_{2 I}\right)^{\prime}$ be the matrices of demeaned instruments stacked across individuals. Let $\mathbf{D}=\left(D_{1}, \ldots, D_{I}\right)^{\prime}$ be the stacked values of the choice variable $D_{i}$. Under random sampling, and the assumptions of Section $2, \frac{\tilde{\mathbf{J}}_{1}^{\prime} \mathbf{D}}{I} \stackrel{p}{\rightarrow} \omega_{1}$ and $\frac{\tilde{\mathbf{J}}_{2}^{\prime} \mathbf{D}}{I} \stackrel{p}{\rightarrow} \omega_{2}$ for some finite constants $\omega_{1}$ and $\omega_{2}$. Under $H_{A}^{I V}: \beta_{I V, 1}-\beta_{I V, 2}=\left[\int_{0}^{1} M T E\left(u_{D}\right)\left(h_{1}\left(u_{D}\right)-h_{2}\left(u_{D}\right)\right) d u_{D}\right] / \sqrt{I}$, the noncentrality parameter of the chi-square distribution of the test statistic is

$$
\lambda_{\mathrm{TV}, 1,2}=\frac{1}{2}\left(\int_{0}^{1} \operatorname{MTE}\left(u_{D}\right)\left(h_{1}\left(u_{D}\right)-h_{2}\left(u_{D}\right)\right) d u_{D}\right)^{2} \Psi_{1,2}^{-1}
$$

where 


$$
\begin{aligned}
\Psi_{1,2}= & \left(\alpha^{2}\right)\left[\frac{\operatorname{Var}\left(J_{1}\right)}{\omega_{1}^{2}}-\frac{2 \operatorname{Cov}\left(J_{1}, J_{2}\right)}{\omega_{1}\left(\omega_{2}\right.}+\frac{\operatorname{Var}\left(J_{2}\right)}{\omega_{2}^{2}}\right] \\
& +\int_{0}^{1}\left[2 E\left(\alpha \beta \mid U_{D}=u_{D}\right)+E\left(\beta^{2} \mid U_{D}=u_{D}\right)\right] h_{\Omega_{2} J_{1}, J_{2}}\left(u_{D}\right) d u_{D} \\
& -\left[\int_{0}^{1} \operatorname{MTE}\left(u_{D}\right)\left(h_{1}\left(u_{D}\right)-h_{2}\left(u_{D}\right)\right) d u_{D}\right]^{2} .
\end{aligned}
$$

Defining $J_{1}^{*}=J_{1}-E\left(J_{1}\right)$ and $J_{2}^{*}=J_{2}-E\left(J_{2}\right)$, the weight $h_{\Omega J_{1}, J_{2}}(\cdot)$ is given by

$$
h_{\Omega, J_{1}, J_{2}}\left(u_{D}\right)=\int_{u_{D}}^{1} \int_{-\infty}^{\infty}\left(\frac{J_{1}^{*}}{\omega_{1}}-\frac{J_{2}^{*}}{\omega_{2}}\right)^{2} f_{\left(J_{1}-J_{2}\right), P}\left(j_{1}-j_{2}, P(z)\right) d\left(j_{1}-j_{2}\right) d P(z)=E\left[\left(\frac{J_{1}^{*}}{\omega_{1}}-\frac{J_{2}^{*}}{\omega_{2}}\right)^{2} \mid P(Z)>u_{D}\right] \operatorname{Pr}\left(P(Z)>u_{D}\right) .
$$

20 The derivation follows a logic similar to that used to derive (7).21 Notice that not only will the difference in the IV estimands depend on the alternative under consideration, but the variance of the difference between the IV estimators will also depend on the alternative under consideration.

We present this characterization of the variance in order to understand the properties of tests of $H_{0}$ based on IV estimators. This expression for the variance is not meant as a guide for how to implement such tests. In practice the analyst would form the test statistic using a standard estimator of the variance of the vector of IV estimates.

In general, the weights presented above do not have simple analytical expressions. They do in the case of a model with normal error terms with normally distributed instruments and a linear index structure for the choice equation. However, for this case, the proposed IV test has no power, because, and as previously discussed and as established in Appendix B, $\beta_{\mathrm{IV}, \mathrm{J}_{1}}$ $\equiv \beta_{\mathrm{IV}, \mathrm{J}_{2}}$ irrespective of the truth or falsity of $H_{0}$. For this case, the noncentrality parameter of the asymptotic chi-square distribution of the test statistic will be zero so the power of the test equals its size. To have a test with any power, we have to rule out instruments with equal weights. Since the weights can be constructed from the data on $Z$, it is possible to check this condition in any sample. ${ }^{22}$

We do not formally analyze conditions that guarantee that the two instruments $J_{1}$ and $J_{2}$, constructed from $Z$, optimize the power function of the test. From the expression for the noncentrality parameter, one can see the ingredients required to construct an asymptotically most powerful test. Let $Z \in \mathbb{R}^{k}$ be the vector of available instruments and let $\mathcal{J}=\left\{J \mid J: \mathbb{R}^{k} \rightarrow \mathbb{R}\right\}$ be the space of functions which map the vector of instruments to the real line. Then for a given MTE, the optimal choice of $J_{1}$ and $J_{2}$ solves the problem

$$
\max _{J_{1} \in \mathcal{T}, J_{2} \in \mathcal{J}} \frac{1}{2}\left(\int_{0}^{1} \operatorname{MTE}\left(u_{D}\right)\left(h_{1}\left(u_{D}\right)-h_{2}\left(u_{D}\right)\right) d u_{D}\right)^{2} \Psi_{1,2}^{-1}
$$

The optimal choice of instruments will generally depend on the shape of the $\operatorname{MTE}\left(u_{D}\right){ }^{23}$

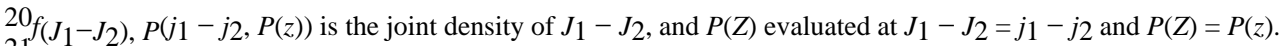

${ }^{21}$ The logic is not, however, identical. Using $\left(J_{1}-J_{2}\right)$ as an instrument and testing if $\beta_{\mathrm{IV}}, J_{1}-J_{2}=0$ is not equivalent to the test presented in the main text of the paper. The denominators of the IVs differ in the two approaches.

${ }^{22}$ It would be desirable to develop a formal test for equality of the two IV weights. The required ingredients are in the literature. We leave the formal derivation for another occasion. 
We present an example with two non-normal instruments in Figure 4. Specifically, let $D=$ $\mathbf{1}\left(\gamma_{1} Z_{1}+\gamma_{2} Z_{2}>V\right)$ where the vector $Z=\left(Z_{1}, Z_{2}\right)$ is distributed as a multivariate mixture of normals with the distribution given at the base of the figure. The unobservables are assumed to be generated by a normal generalized Roy model. The test of equality of the IV estimators constructed using these two instruments has power to detect deviations from $H_{0}$. Figure 4A plots the weights $h_{1}(\cdot)$ and $h_{2}(\cdot)$ which the IV estimator places on the MTE, using $Z_{1}$ or $Z_{2}$ respectively. The weights must differ for the test based on the difference in IV estimators to have power to detect deviations from $H_{0}$. When the mixing proportion in the mixture of normals is 0.45 , the instruments are highly non-normal and the IV weights differ substantially. However, when the mixing proportion is 0.75 , the instruments become closer to normal, the weights become very similar, and the test of $H_{0}$ loses power. This case is discussed further in Heckman, Schmierer, and Urzua (2010).

Another example of a test that has power to detect deviations from $H_{0}$, even with normal instruments, constructs IV estimators using nonlinear functions of $Z$. We consider a normal generalized Roy model where there is one $Z$ variable in the choice equation that is normally distributed, $D=\mathbf{1}(Z>V)$. We plot the weights of the IV estimators based on $Z$ and $Z^{2}$.

Figure 4B plots the weights for these two choices of instruments. The weights differ, and in addition the amount by which they differ generally depends on the distribution of $Z$. We plot the weights for two choices of the mean of $Z$ presented in the figure. These choices clearly affect the weights and hence will generally affect the power of a test of $H_{0}$ based on these IV estimators.

Another choice of instruments uses $P(Z)$ on disjoint intervals of the support of $P(Z)$ as two instruments. Form two disjoint intervals $\left[p_{1}, p^{-}\right]$and $\left[p_{2}, p_{2}^{-}\right]$, and construct IV estimators over these intervals as sample analogs to

$$
\beta_{I V\left[p_{-1}, \bar{p}_{1}\right]}=\frac{\operatorname{Cov}\left(Y, P(Z) \mid P(Z) \in\left[\begin{array}{c}
\left.\left.p, \bar{p}_{1}\right]\right) \\
-1
\end{array}\right]\right.}{\operatorname{Var}\left(P(Z) \mid P(Z) \in\left[{ }_{-1}^{p}, \bar{p}_{1}\right]\right)}
$$

and

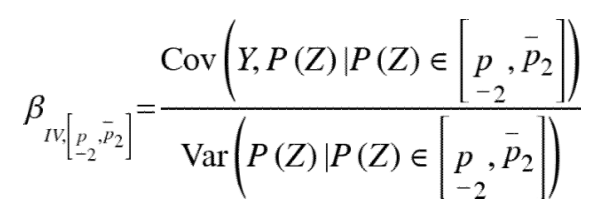

and test

$$
\begin{gathered}
H_{0}^{I V}: \beta_{I V\left[p_{-1}, \bar{p}_{1}\right]}=\beta_{I V\left[\left[p_{-2}, \bar{p}_{2}\right]\right.} \\
H_{A}^{I V}: \beta_{I V\left[p_{-1}, \bar{p}_{1}\right]} \neq \beta_{I V\left[{ }_{-2}, \bar{p}_{2}\right]}
\end{gathered}
$$

\footnotetext{
${ }^{23}$ More generally, one could use multiple instruments and base a test on multiple contrasts of the set of instruments. We do not develop this test in this paper.
} 
There is no a priori guidance on which intervals to use so we consider two ways to construct intervals over which to form IV estimates: (1) use the intervals $\left[0, p_{m e d}\right)$ and $\left[p_{m e d}, 1\right]$ where $p_{m e d}$ is the sample median of $P(Z)$, and (2) use the intervals $\left[0, p_{q 1}\right),\left[p_{q 1}, p_{q 2}\right),\left[p_{q 2}, p_{q 3}\right)$ and $\left[p_{q 3}, 1\right]$, where $p_{q j}$ is the $j$ th sample quartile of the distribution of $P(Z)$ and form all pairwise contrasts between these estimates. Note that even though we split the propensity score into four intervals, we are still conducting pairwise tests. However, because there is a multiplicity of pairwise tests, we must control the size of the test. We do this by using the stepdown procedure of Romano and Wolf (2005). Figures 4C and 4D plot the weights for the instruments constructed in this manner. These weights are nonoverlapping by construction and will also depend on the distribution of the instrument $Z$.

The power of the test of $H_{0}$ based on IV estimators also depends on the variance (12), which determines the denominator of the noncentrality parameter. The important terms which are affected by the choice of instruments are the variance of the difference in the instruments $\left[\frac{\operatorname{Var}\left(J_{1}\right)}{\omega_{1}^{2}}-\frac{2 \operatorname{Cov}\left(J_{1}, J_{2}\right)}{\omega_{1} \omega_{2}}+\frac{\operatorname{Var}\left(J_{2}\right)}{\omega_{2}^{2}}\right]$ and the variance weight $h_{\Omega J_{1}, J_{2}}(\cdot)$. The variance of the difference in the instruments is identified from the distribution of $Z$ given $X$. The weights $h_{\Omega J_{1}, J_{2}}(\cdot)$, can also be estimated from the data but are less transparent. For each of the examples presented in Figure 4, we plot the variance weights $h_{\Omega J_{1}, J_{2}}(\cdot)$. In the case of the normal generalized Roy model, the weights are more intuitive and more easily calculated when conditioning directly on $V=v$ (rather than $U_{D}=u_{D}$ ), so we plot them as a function of $v$. Figure 5 plots the variance weights. Ceteris paribus, the larger the variance weights, the larger is the variance of the difference in the IV estimators and hence the lower the power of a test based on this difference. In Panel A of Figure 5 we see that when the mixing proportion is 0.45 the variance of the difference in the estimators is higher than when the mixing proportion is 0.75 due to the fact that the IV weights covary highly when the instruments are closer to normal so the variance of their difference is smaller. In Panel B, the variance weights are roughly similar for $E(Z)=1$ and $E(Z)=-0.5$. Finally, in Panel C the variance weights are much larger when $E(Z)=1$ than when $E(Z)=0$. This demonstrates that even when the IV weights are nonoverlapping, as is the case in both examples in Panel C, the variance of the difference in the IV estimators will generally depend on the distribution of $Z$.

We emphasize that the specific comparisons of IV estimators presented in this section are illustrative examples. Our formal analysis is completely general and allows for any choice of valid instruments which satisfy (A-1)-(A-5).

\section{Testing $H_{0}$ by Testing for Linearity}

We next consider tests of $H_{0}$ based on linearity in $p$. Keeping the conditioning on $X$ implicit, we can write (3) as

$$
E(Y \mid P(Z)=p)=\mu+g(p)
$$

for some general nonlinear function $g(\cdot)$ where $\mu$ and $g$ may depend on $X$. Our test for the absence of selection on the gain to treatment is a test of whether the function $g(\cdot)$ belongs to the linear parametric family $\mathcal{F}=\left\{a+b p,(a, b) \in \mathbb{R}^{2}\right\}$. Let $\mathcal{P}$ be the support of $P(Z)$, with typical element $p \in \mathcal{P}$. The null hypothesis of linearity can be written as

$$
H_{0}^{L} \text { :There exists some }(a, b) \in \mathbb{R}^{2} \quad \text { such that } g(p)=a+b p \quad \text { for almost all } p \in \mathcal{P},
$$


while the alternative is

$$
H_{A}^{L} \text { :There exists some }(a, b) \in \mathbb{R}^{2} \text { such that } g(p)=a+b p \text { for almost all } p \in \mathcal{P} \text {. }
$$

There is a large and still unsettled literature in econometrics and statistics dealing with specification tests of this type. ${ }^{24}$ These tests proceed in one of two ways: (i) testing orthogonality restrictions implied by the parametric model, or (ii) comparing a nonparametric estimate of $g(p)$ with a parametric estimate, $\hat{a}+\hat{b p}$. We discuss both types of tests. Heckman, Schmierer, and Urzua (2010) present Monte Carlo analyses of the power of these tests. We briefly discuss a third test due to Li and Nie (2007).

\section{Linearity Test 1: Wald Test Based on Series}

The first test of linearity of $E(Y \mid P(Z)=p)$ in $p$ determines whether terms in addition to $p$ are required to fit the data. It is instructive to consider the case of the normal selection model as a baseline. When the data are generated from the normal generalized Roy model, we can characterize $E(Y \mid P(Z)=p)$ by

$$
E(Y \mid P(Z)=p)=\bar{\alpha}+\bar{\beta} p+\tau \int_{0}^{p} \Phi^{-1}\left(u_{D}\right) d u_{D} .
$$

Heckman, Schmierer, and Urzua (2010) examine this case in depth.

In the general non-normal case, polynomials can approximate classes of smooth alternatives for the function $g(\cdot)$. One can estimate $E(Y \mid P(Z)=p)$ using polynomials of degree 2 or higher. Polynomials approximate well a broad class of functions. Exploring power in this class gives us an indication of the power of our procedures against such alternatives. ${ }^{25}$ One specification of a more general, but still parametric, alternative model is

$$
g(P(Z))=\sum_{l=0}^{L} \phi_{l}(P(Z))^{l},
$$

where $L$ is assumed to be known. ${ }^{26}$ The proposed test for linearity is

$$
\begin{aligned}
& H_{0}: \phi_{l}=0 \text { for } l=2, \ldots, L \\
& H_{A}: \phi_{l} \neq 0 \text { for some (or all) } l=2, \ldots, L .
\end{aligned}
$$

Heckman, Schmierer, and Urzua (2010) develop properties of this test for linearity.

\section{Linearity Test 2: Bierens Conditional Moment Test}

One can also test the validity of representation (3) using orthogonality restrictions implied by the parametric model. One approach is the conditional moment (CM) test of Bierens

\footnotetext{
${ }^{24}$ See, e.g., Horowitz and Spokoiny (2001) and the references therein. The properties of particular tests depend on the specification of alternatives.

25 Ichimura and Todd (2007) discuss the properties of series estimators. Newey (1997) establishes convergence rates and proves asymptotic normality of such estimators.

26 Below, we discuss a procedure when $L$ is unknown.
} 
(1990). ${ }^{27}$ This test uses the fact that under the null hypothesis the following moment condition must be satisfied

$$
E\left[Y-a_{0}-b_{0} P(Z) \mid P(Z)\right]=0
$$

for the true parameter vector $\left(a_{0}, b_{0}\right) \in \mathbb{R}^{2}$. This conditional moment restriction implies the set of unconditional moment restrictions

$$
E\left[\left(Y-a_{0}-b_{0} P(Z)\right) \exp \left(t^{\prime} \Lambda(P(Z))\right)\right]=0
$$

for all $t \in \mathbb{R}$, for some bounded one-to-one, mapping from $\mathbb{R}$ into $\mathbb{R}$. A test can be constructed using the sample analog of the left-hand side of (14). Bierens (1990) shows how one can use sample analogs to construct a test statistic which, under the null hypothesis, converges in distribution to a $\chi_{1}^{2}$ and under the alternative diverges to infinity. Heckman, Schmierer, and Urzua (2010) discuss the properties of this test.

\section{The Preceding Tests are Conditional Moment Tests ${ }^{28}$}

All of the tests discussed so far test if

$$
E(Y \mid P(Z))=a+b P(Z)
$$

which is equivalent to

$$
E[h(P(Z))[Y-a-b P(Z)]]=0
$$

for any function $h$. Conditional moment tests would typically use a vector of functions $h(P(z))$ to construct tests.

The tests previously discussed use different choices of $h(P)$. For the Bierens test, one would use $h(P(Z))=\exp \left(t^{\prime} \Lambda(P(Z))\right)$. The test of linearity based on polynomials takes $h(P(Z))=1$, $P(Z),(P(Z))^{2}, \ldots,(P(Z))^{L}$. The IV test can also be cast in this framework, as the following argument shows.

The plim of the IV estimator obtained using $J_{k}(Z), k=1, \ldots, K$, as an instrument are the values of $\left(a_{k}, b_{k}\right)$ that solve

$$
E\left[J_{k}(Z)\left[Y-a_{k}-b_{k} D\right]\right]=0
$$

and

$$
E\left[Y-a_{k}-b_{k} D\right]=0, \quad k=1, \ldots, K
$$

By the law of iterated expectations, this is equivalent to solving

${ }^{27}$ See also Bierens (1982) and Bierens and Ploberger (1997) for related tests. Newey (1985) discusses conditional moment tests more generally.

${ }^{28}$ We thank Edward Vytlacil for suggesting this unifying approach. 


$$
\begin{array}{r}
E\left[J_{k}(Z)\left[E(Y \mid Z)-a_{k}-b_{k} P(Z)\right]\right]=0 \\
E\left[E(Y \mid Z)-a_{k}-b_{k} P(Z)\right]=0,
\end{array}
$$

which is equivalent to solving

$$
\begin{array}{r}
E\left[J_{k}(Z)\left[Y-a_{k}-b_{k} P(Z)\right]\right]=0 \\
E\left[Y-a_{k}-b_{k} P(Z)\right]=0 .
\end{array}
$$

For one instrument there is no test, but for two or more $(K \geq 2)$, one can test if a common pair of $(a, b)$ satisfies all of the moment conditions produced from using different instrumental variables. This is the classical test of overidentification. Thus, all of the tests previously discussed can be viewed as conditional moment tests.

\section{Linearity Test 3: A Semiparametric Test Based on Local Linear Regression 29}

A potential problem with the test based on series estimators (Linearity Test 1 ) is that it assumes that the degree of the highest order polynomial in $P(Z)$ is finite and known. A semiparametric approach that did not rely on strong functional form assumptions about the generator model is more desirable.

$\mathrm{Li}$ and Nie (2007) use local linear regression methods to develop a test for linearity of an unknown parametric function in a semiparametric model. They develop a test of linearity of the unknown nonparametric component (linearity in $P(Z)$ in our setup) that can be applied to the problem analyzed in this paper if it is adapted to the case of an estimated $P(Z)$. If $P(Z)$ is parametric and its coefficients are $\sqrt{N}$ estimable, their analysis can be applied directly. The case where $P(Z)$ is estimated nonparametrically is left for another occasion.

Li and Nie (2007) conduct a Monte Carlo study of their approach. They show good size and power properties for their test statistic. Their test can be interpreted as a local conditional moment test.

\section{Conditioning on $X$}

Throughout, we have conditioned on $X$. An important practical problem not addressed in this paper but common to all empirical models is picking the appropriate conditioning set, and determining how to explicitly model the dependence of $Y$ on $X$. Heckman, Schmierer, and Urzua (2010) discuss the power of these tests and conduct extensive Monte Carlo studies.

\section{Summary and Conclusion}

P.A.V.B Swamy's classic work $(1971,1974)$ developed estimators for the uncorrelated random coefficient model. This is the case when $H_{0}$ is true. In this paper, building on the work of Heckman and Vytlacil (1999,2005,2007a,b) and Heckman, Schmierer, and Urzua (2010), we develop tests for the presence of a correlated random coefficient model and related tests on parameters derived from the model. All of the tests we consider can be interpreted as conditional moment tests. We develop instrumental variable tests for the null hypothesis of the absence of a correlated random coefficient model. To implement it, we develop the sampling distribution of the IV estimator using the marginal treatment effect and

\footnotetext{
${ }^{29}$ We thank Xiaohong Chen for directing us to this paper and clarifying our thinking about semiparametric approaches to testing for linearity.
} 
its extensions to higher moments of the distribution of the heterogeneity on which agents select.

Heckman, Schmierer, and Urzua (2010) conduct a Monte Carlo investigation of the power of these tests. One disturbing finding from their work is that the power of all of the tests we consider is low. They show that among all of the tests considered, the test based on comparing alternative IV estimators above and below the median propensity score has the highest power.

This paper analyzes the case of a binary treatment. Heckman, Urzua, and Vytlacil (2006) and Heckman and Vytlacil (2007b) analyze the cases of a multiple treatment model generated by an ordered choice model with stochastic thresholds and a multiple treatment model generated by an unordered choice model. In all of these cases, IV produces an instrument-dependent parameter so the IV test for selection on unobserved gains based on comparing the estimands of two different IVs developed in this paper carries over in general to these settings. A test of linearity of the conditional expectation of $Y$ given $P$ in (a vector of) $P$ is developed for the outcome model for multiple treatments generated by the ordered choice model in Heckman, Urzua, and Vytlacil (2006). It also applies to the unordered multiple choice model that identifies the treatment effect of a gain compared to the next best option which Heckman, Urzua and Vytlacil show is a direct extension of the binary model.

\section{Acknowledgments}

This research was supported by NIH R01-HD043411, NSF SES-024158, the American Bar Foundation and the Geary Institute, University College Dublin, Ireland. The views expressed in this paper are those of the authors and not necessarily those of the funders listed here. We have received helpful comments from Pedro Carneiro, Jeremy Fox, Joel Horowitz, Benjamin Moll, Azeem Shaikh, Christopher Taber, Edward Vytlacil, and Sergio Urzua.

\section{Appendix}

\section{A The Variance of Linear IV in the Correlated Random Coefficient Model}

The IV estimator, using instrument $J(Z)$, is

$$
\widehat{\beta}_{I V, J}=\frac{\sum Y_{i} \tilde{J}_{i}}{\sum \tilde{D}_{i} \tilde{J}_{i}}
$$

and hence

$$
\sqrt{I \beta_{I V, J}}=\frac{\frac{1}{\sqrt{I}} \sum\left(J_{i}-\bar{J}\right)\left(\alpha_{i}+\beta_{i} D_{i}\right)}{\frac{1}{I} \sum D_{i} \widetilde{J}_{i}} .
$$

Invoking standard central limit theorems,

$$
\sqrt{I}\left(\widehat{\beta}_{I V, J}-\beta_{I V, I}\right) \stackrel{d}{\rightarrow} N\left(0, \Omega_{J}\right)
$$

Defining $J^{*}=J-E(J)$, where $\Omega_{J}$ is given by 


$$
\begin{aligned}
& \Omega_{J}=E\left\{\left[\frac{Y J^{*}}{\omega_{J}}-E\left(\frac{Y J^{*}}{\omega_{J}}\right)\right]^{2}\right\} \\
&=E\left[\frac{Y^{2}\left(J^{*}\right)^{2}}{\omega_{J}^{2}}\right] \\
&-\left(E\left[\frac{Y J^{*}}{\omega_{J}}\right]\right)^{2} \\
&= E\left[\frac{Y^{2}\left(J^{*}\right)^{2}}{\omega_{J}^{2}}\right] \\
&-\left(\int_{0}^{1} E\left(\beta \mid U_{D}=u_{D}\right) h_{J}\left(u_{D}\right) d u_{D}\right)^{2} \\
&= \frac{1}{\omega_{J}^{2}} E\left[(\alpha+\beta D)^{2}\left(J^{*}\right)^{2}\right] \\
&-\left(\int_{0}^{1} E\left(\beta \mid U_{D}=u_{D}\right) h_{J}\left(u_{D}\right) d u_{D}\right)^{2} \\
&=\frac{1}{\omega_{J}^{2}} E\left[\alpha^{2}\left(J^{*}\right)^{2}\right] \\
&+\frac{2}{\omega_{J}^{2}} E\left[\alpha \beta D\left(J^{*}\right)^{2}\right] \\
&+\frac{1}{\omega_{J}^{2}} E\left[\beta^{2} D\left(J^{*}\right)^{2}\right] \\
&-\left(\int_{0}^{1} E\left(\beta \mid U_{D}=u_{D}\right) h_{J}\left(u_{D}\right) d u_{D}\right)^{2} .
\end{aligned}
$$

Using the law of iterated expectations as well as the assumption that $\alpha$ is independent of $Z$, this expression can be written as

$$
\Omega_{J}=E\left[\alpha^{2}\right] \frac{\operatorname{Var}(J)}{\omega_{J}^{2}}+\frac{1}{\omega_{J}^{2}} 2 E\left[\alpha \beta\left(J^{*}\right)^{2} \mid D=1\right] \operatorname{Pr}(D=1)+\frac{1}{\omega_{J}^{2}} E\left[\beta^{2}\left(J^{*}\right)^{2} \mid D=1\right] \operatorname{Pr}(D=1)-\left(\int_{0}^{1} E\left(\beta \mid U_{D}=u_{D}\right) h_{J}\left(u_{D}\right) d u_{D}\right)^{2}
$$

where

$$
h_{J}\left(u_{D}\right)=\frac{E\left[J^{*} \mid P(Z)>u_{D}\right] \operatorname{Pr}\left(P(Z)>u_{D}\right)}{\omega_{J}} .
$$

Under the conditions of Fubini's Theorem, we can exchange the order of integration and write 


$$
\begin{aligned}
& \Omega_{J}=E\left[\alpha^{2}\right] \frac{\operatorname{Var}(J)}{\omega_{J}^{2}} \\
&+\int_{0}^{1}\left[2 E\left(\alpha \beta \mid U_{D}=u_{D}\right)+E\left(\beta^{2} \mid U_{D}=u_{D}\right)\right] \frac{E\left(\left(J^{*}\right)^{2} \mid P(Z)>u_{D}\right) \operatorname{Pr}\left(P(Z)>u_{D}\right)}{\omega_{J}^{2}} d u_{D} \\
&-\left(\int_{0}^{1} E\left(\beta \mid U_{D}=u_{D}\right) h_{J}\left(u_{D}\right) d u_{D}\right)^{2} \\
&=E\left[\alpha^{2}\right] \frac{\operatorname{Var}(J)}{\omega_{J}^{2}} \\
&+\int_{0}^{1}\left[2 E\left(\alpha \beta \mid U_{D}=u_{D}\right)+E\left(\beta^{2} \mid U_{D}=u_{D}\right)\right] h_{\Omega_{J}}\left(u_{D}\right) d u_{D} \\
&-\left(\int_{0}^{1} E\left(\beta \mid U_{D}=u_{D}\right) h_{J}\left(u_{D}\right) d u_{D}\right)^{2}
\end{aligned}
$$

where

$h_{\Omega_{J}}\left(u_{D}\right)=\frac{1}{\omega_{J}^{2}} \int_{-\infty}^{\infty}(j-E(J))^{2} \int_{u_{D}}^{1} f_{P, J}(P(z), j) d P(z) d j=\frac{\left.E\left[(J-E(J))^{2} \mid P(Z)>u_{D}\right)\right] \operatorname{Pr}\left(P(Z)>u_{D}\right)}{\omega_{J}^{2}}$

which is the expression in the text.

\section{Appendix}

\section{B Proof of Invariance of the IV Estimand to the Choice of a Linear Instrument under Normality with a Linear Index Choice Equation}

Suppose that the choice equation has a linear index structure, so that

$$
D=1(Z \gamma>V)
$$

where $Z \sim N\left(Z^{-}, \Sigma_{Z}\right)$, an $L$-dimensional multivariate normal random variable, $\gamma$ an $L \times 1$ vector and $V \sim N\left(0, \sigma_{V}^{2}\right)$. Consider the instrument $J(Z)$, which is a linear function of $Z$, say $Z^{\prime} \eta$. In this case, the IV estimand (written as a weighted average over the support of $V$ ) is

$$
\beta_{I V, J}=\int_{-\infty}^{\infty} \operatorname{MTE}(v) h_{J}(v) \phi\left(\frac{v}{\sigma_{v}}\right) d v
$$

where $\phi(\cdot)$ is a standard normal pdf and the IV weight is

$$
h_{J}(v)=\frac{E[J(Z)-E(J(Z)) \mid Z \gamma>v] \operatorname{Pr}(Z \gamma>v)}{\operatorname{Cov}(J(Z), D)} .
$$

Under the assumption of multivariate normality for the instruments, 


$$
h_{J}(v)=\frac{\frac{\operatorname{Cov}(J(Z), Z \gamma)}{\sqrt{\operatorname{Var}(Z \gamma)}} \phi\left(\frac{v-\bar{Z} \gamma}{\sqrt{\operatorname{Var}(Z \gamma)}}\right)}{\frac{\operatorname{Cov}(J(Z), Z \gamma-V)}{\sqrt{\operatorname{Var}(Z \gamma-V)}} \phi\left(\frac{-\bar{Z} \gamma}{\sqrt{\operatorname{Var}(Z \gamma-V)}}\right)} .
$$

Under assumption (A-1) in Section 2, $\operatorname{Cov}(J(Z), Z \gamma)=\operatorname{Cov}(J(Z), Z \gamma-V)$, and we obtain

$$
h_{J}(v)=\frac{\frac{1}{\sqrt{\operatorname{Var}(Z \gamma)}} \phi\left(\frac{v-\bar{Z} \gamma}{\sqrt{\operatorname{Var}(Z \gamma)}}\right)}{\frac{1}{\sqrt{\operatorname{Var}(Z \gamma-V)}} \phi\left(\frac{-\bar{Z} \gamma}{\sqrt{\operatorname{Var}(Z \gamma-V)}}\right)} .
$$

That is, the IV weights, and hence the IV estimand, are the same for all $J(Z)=Z$ ' $\eta$ for any $\eta$.

\section{References}

Abbring, JH.; Heckman, JJ. Econometric evaluation of social programs, part III: Distributional treatment effects, dynamic treatment effects, dynamic discrete choice, and general equilibrium policy evaluation. In: Heckman, J.; Leamer, E., editors. Handbook of Econometrics. Vol. 6B. Elsevier; Amsterdam: 2007. p. 5145-5303.

Ai C, Chen X. Efficient estimation of models with conditional moment restrictions containing unknown functions. Econometrica 2003;71(6):1795-1843.

Bierens HJ. Consistent model specification tests. Journal of Econometrics 1982;20(1):105-134.

Bierens HJ. A consistent conditional moment test of functional form. Econometrica November;1990 58(6):1443-1458.

Bierens HJ, Ploberger W. Asymptotic theory of integrated conditional moment tests. Econometrica September;1997 65(5):1129-1151.

Björklund A, Moffitt R. The estimation of wage gains and welfare gains in self-selection. Review of Economics and Statistics February;1987 69(1):42-49.

Griliches Z. Estimating the returns to schooling: Some econometric problems. Econometrica January; 1977 45(1):1-22. [PubMed: 12278790]

Heckman JJ. Micro data, heterogeneity, and the evaluation of public policy: Nobel lecture. Journal of Political Economy August;2001 109(4):673-748.

Heckman, JJ.; Robb, R. Alternative methods for evaluating the impact of interventions. In: Heckman, J.; Singer, B., editors. Longitudinal Analysis of Labor Market Data. Vol. 10. Cambridge University Press; New York: 1985. p. 156-245.

Heckman JJ, Schmierer D, Urzua S. Testing the correlated random coefficient model. Journal of Econometrics. 2010 Forthcoming.

Heckman JJ, Urzua S, Vytlacil EJ. Understanding instrumental variables in models with essential heterogeneity. Review of Economics and Statistics 2006;88(3):389-432.

Heckman JJ, Vytlacil EJ. Instrumental variables methods for the correlated random coefficient model: Estimating the average rate of return to schooling when the return is correlated with schooling. Journal of Human Resources 1998 Fall;33(4):974-987.

Heckman JJ, Vytlacil EJ. Local instrumental variables and latent variable models for identifying and bounding treatment effects. Proceedings of the National Academy of Sciences April;1999 96(8): 4730-4734.

Heckman, JJ.; Vytlacil, EJ. In: Hsiao, C.; Morimune, K.; Powell, JL., editors. Local instrumental variables; Nonlinear Statistical Modeling: Proceedings of the Thirteenth International Symposium in Economic Theory and Econometrics: Essays in Honor of Takeshi Amemiya; New York: Cambridge University Press. 2001; p. 1-46. 
Heckman JJ, Vytlacil EJ. Structural equations, treatment effects and econometric policy evaluation. Econometrica May;2005 73(3):669-738.

Heckman, JJ.; Vytlacil, EJ. Econometric evaluation of social programs, part I: Causal models, structural models and econometric policy evaluation. In: Heckman, J.; Leamer, E., editors. Handbook of Econometrics. Vol. 6B. Elsevier; Amsterdam: 2007a. p. 4779-4874.

Heckman, JJ.; Vytlacil, EJ. Econometric evaluation of social programs, part II: Using the marginal treatment effect to organize alternative economic estimators to evaluate social programs and to forecast their effects in new environments. In: Heckman, J.; Leamer, E., editors. Handbook of Econometrics. Vol. 6B. Elsevier; Amsterdam: 2007b. p. 4875-5144.

Horowitz JL, Spokoiny VG. An adaptive, rate-optimal test of a parametric mean-regression model against a nonparametric alternative. Econometrica May;2001 69(3):599-631.

Ichimura, H.; Todd, PE. Implementing nonparametric and semiparametric estimators. In: Heckman, J.; Leamer, E., editors. Handbook of Econometrics. Vol. 6B. Elsevier; Amsterdam: 2007.

Imbens GW, Angrist JD. Identification and estimation of local average treatment effects. Econometrica March;1994 62(2):467-475.

Li R, Nie L. Efficient statistical inference procedures for partially nonlinear models and their applications. Biometrics November;2007 64(3):904-911. [PubMed: 18047529]

McFadden, D. Conditional logit analysis of qualitative choice behavior. In: Zarembka, P., editor. Frontiers in Econometrics. Academic Press; New York: 1974.

McFadden, D. Econometric models of probabilistic choice. In: Manski, C.; McFadden, D., editors. Structural Analysis of Discrete Data with Econometric Applications. MIT Press; Cambridge, MA: 1981.

Mincer, J. Schooling, Experience and Earnings. Columbia University Press for National Bureau of Economic Research; New York: 1974.

Newey WK. Maximum likelihood specification testing and conditional moment tests. Econometrica September;1985 53(5):1047-1070.

Newey WK. Convergence rates and asymptotic normality for series estimators. Journal of Econometrics July;1997 79(1):147-168.

Quandt RE. The estimation of the parameters of a linear regression system obeying two separate regimes. Journal of the American Statistical Association December;1958 53(284):873-880.

Romano JP, Wolf M. Exact and approximate stepdown methods for multiple hypothesis testing. Journal of the American Statistical Association March;2005 100(469):94-108.

Swamy, P. Statistical Inference in Random Coefficient Regression Models. Springer-Verlag; New York: 1971.

Swamy, P. Linear models with random coefficients. In: Zarembka, P., editor. Frontier in Econometrics. Academic Press; New York: 1974.

Thurstone LL. A law of comparative judgement. Psychological Review 1927;34:273-286.

Vytlacil EJ. Independence, monotonicity, and latent index models: An equivalence result. Econometrica January;2002 70(1):331-341.

Yitzhaki, S. On using linear regression in welfare economics. Department of Economics, Hebrew University; 1989. Working Paper 217 

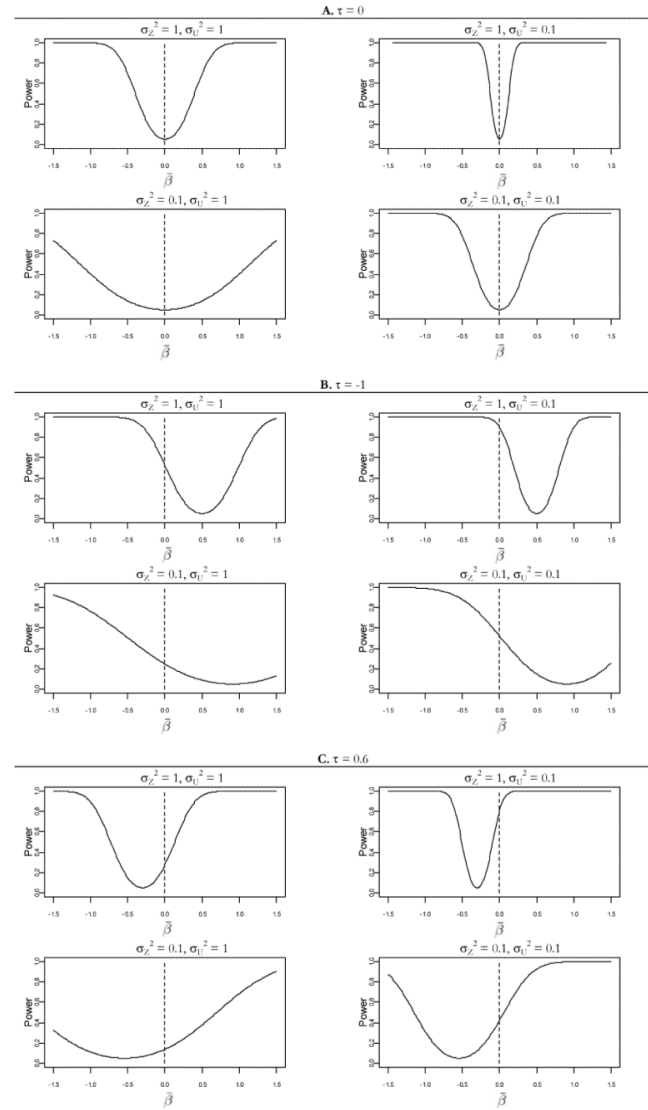

Figure 1.

Power function for a Wald test of $\beta^{-}=0$ based on the sampling distribution of $\hat{\beta}_{I V, J}$.

Note: Each plot shows the power with a hypothetical sample size of 500. The size of the test is 0.05 . The model is the normal generalized Roy model with the unobservables jointly normal with variance $\sigma_{V}^{2}$ and correlation 0.5 . The choice equation is $D=\mathbf{1}(Z>V)$ where $V \sim$ $N(0,1)$ and $Z \sim N\left(1, \sigma_{Z}^{2}\right)$. The power functions plot the power of the Wald test of $\beta_{I V, J}=0$ for alternative values of $\beta^{-}$. The vertical dashed lines denote the null hypothesis $\beta^{-}=0$. Each panel fixes $\tau=\operatorname{Cov}(\beta, V) / \operatorname{Var}(V)$ at a different level. When $\tau=0, \operatorname{plim} \hat{\beta}_{I V}=\beta_{0}^{-}$which in these figures is zero, and hence the test is consistent. For all nonzero values of $\tau$, the test is inconsistent. 


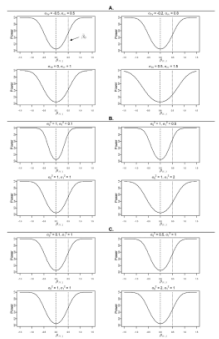

Figure 2.

Power function for the test of the hypothesis that $\operatorname{plim} \hat{\beta}_{I V, J}=0$ when $\beta_{0}^{-}=0.5$. Alternatives are different values of $\beta_{I V, J}^{-}$obtained by fixing $\mathrm{Y}_{J}$ and varying $\beta^{-}$.

Note: Each plot shows the power with a hypothetical sample size of 500. The size of the test is 0.05 . The instrument is normally distributed, $Z \sim N(1,1) ; D=\mathbf{1}(Z>V)$. In panel A, the unobservables are generated with covariances given in the figure and $\sigma_{V}^{2}=1, \sigma_{10}=0, \sigma_{1}^{2}=1$, $\sigma_{0}^{2}=1$. In panels $\mathrm{B}$ and $\mathrm{C}$ the unobservables are generated with variances given in the figure and $\sigma_{V}^{2}=1, \sigma_{10}=0, \sigma_{1 V}=-0.5, \sigma_{0 V}=0.5$. In all panels, under the null hypothesis $\beta_{0}^{-}=0.5$, and alternative hypotheses are generated by changing $\beta^{-}$. The vertical dashed line shows the value of plim $\hat{\beta}_{I V, J}=0$ under the null hypothesis and the vertical dotted line shows the value of $\beta^{-}$under the null hypothesis. 

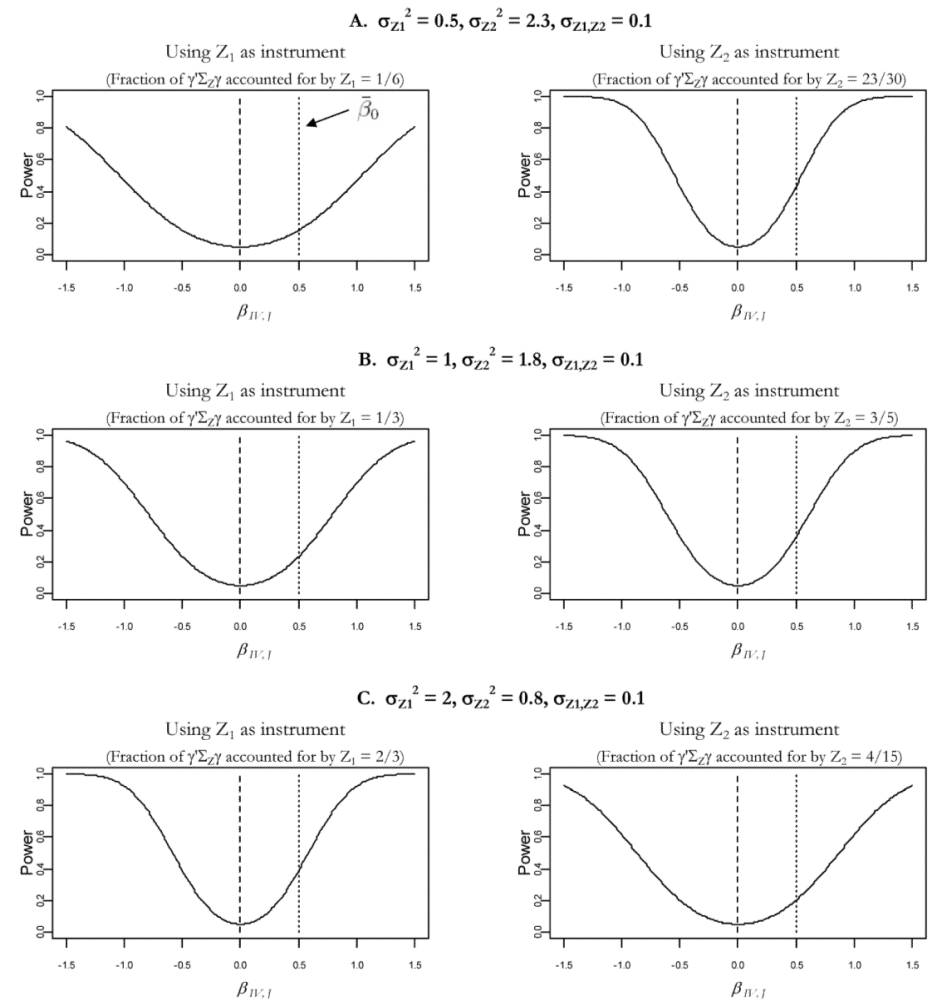

Figure 3.

Power functions for the test of the hypothesis that $\beta_{I V, J}^{-}=\operatorname{plim} \hat{\beta}_{I V, J}=0$ for $\beta_{0}^{-}=0.5$.

Alternatives are different values of $\beta^{-}{ }_{I V, J}$ obtained by fixing $\mathrm{Y}_{J}$ and varying $\beta^{-}$.

Note: Each plot shows the power with a hypothetical sample size of 500 varying $\beta^{-}$keeping $\mathrm{Y}_{J}$ fixed. The size of the test is 0.05 The instruments are distributed normally,

$Z_{1} \sim N\left(1, \sigma_{Z_{1}}^{2}\right), Z_{2} \sim N\left(1, \sigma_{Z_{2}}^{2}\right)$ and $\operatorname{Cov}\left(Z_{1}, Z_{2}\right)=\sigma Z_{1}, Z_{2}, Z_{2}=0.1 ; D=\mathbf{1}\left(Z_{1}+Z_{2}>V\right)$ so $\gamma$ $=(1,1)$. The distribution of the index is held fixed and is distributed $N(2,3)$. The

unobservables are jointly normally distributed with and $\sigma_{v}^{2}=1, \sigma_{10}=0.5, \sigma_{1}^{2}=1, \sigma_{0}^{2}=1, \sigma_{1 V}=$ $-0.3, \sigma_{0 V}=0.3$. In all panels, under the null hypothesis $\beta_{0}^{-}=0.5$, and alternative hypotheses are generated by changing $\beta^{-}$. The vertical dashed line shows the value of $\beta_{I V, J}$ under the null hypothesis and the vertical dotted line shows the value of $\beta^{-}=\beta_{0}^{-}$under the null hypothesis being considered, i.e. that $\beta_{I V, J}=\beta^{-}+\mathrm{Y}_{J}$. 

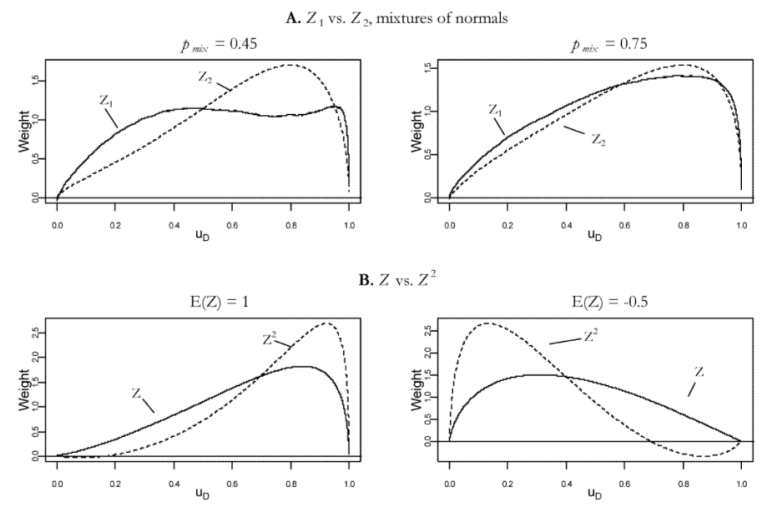

B. $Z$ vs. $Z^{2}$
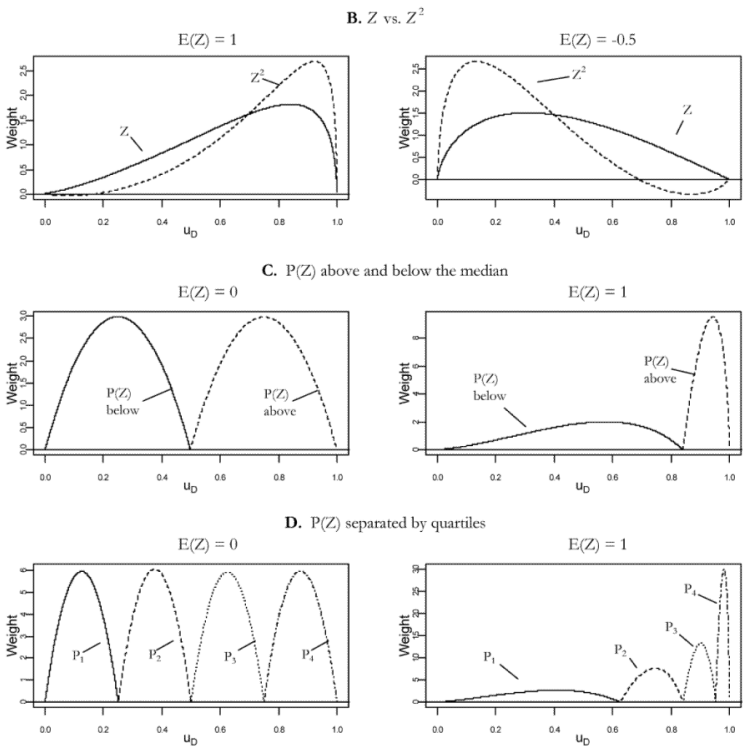

Figure 4.

IV weights for alternative choices of the instrument.

Note: Panel A plots the weights of IV estimates constructed using either $Z_{1}$ or $Z_{2}$ as an instrument where $\left(Z_{1}, Z_{2}\right)$ is distributed as a multivariate mixture of normals, with $D=$ $\mathbf{1}\left(\gamma_{1} Z_{1}+\gamma_{2} Z_{2}>V\right)$. To construct these results, we assume

$$
\left(\begin{array}{l}
Z_{1} \\
Z_{2}
\end{array}\right) \sim p_{\text {mix }} \times N\left(\begin{array}{c}
-0.8 \\
1
\end{array},\left(\begin{array}{cc}
1.4 & 0.5 \\
0.5 & 1.4
\end{array}\right)\right)+\left(1-p_{\text {mix }}\right) \times N\left(\begin{array}{c}
-0.8 \\
1
\end{array},\left(\begin{array}{cc}
0.6 & -0.3 \\
-0.3 & 0.6
\end{array}\right)\right)
$$

and the coefficients in the choice equation are $\gamma_{1}=0.2, \gamma_{2}=1$. In the left plot of Panel A we let $p_{\text {mix }}=0.45$ and in the right plot $p_{m i x}=0.75$. Panel B plots the weights of IV estimates constructed using either $Z$ or $Z^{2}$ as instrument where $Z \sim N\left(\mu_{Z}, 1\right), \mu_{Z}=1$ or $\mu_{Z}=-0.5$, and $D=\mathbf{1}(Z>V)$. Panel $C$ plots the weights of IV estimates constructed using either $P(Z)$ below the median or $P(Z)$ above the median as instruments. Panel D plots the weights of IV estimates constructed using $P(Z)$ in different quartiles of its distribution as instruments. In Panels $\mathrm{C}$ and $\mathrm{D} Z \sim N\left(\mu_{Z}, 1\right), \mu_{Z}=0$ or $\mu_{Z}=1$, and $D=\mathbf{1}(Z>V)$. In all of the plots, we set $\sigma_{V}^{2}=1$. 
A. $Z_{1}$ vs. $Z_{2}$, mixtures of normals
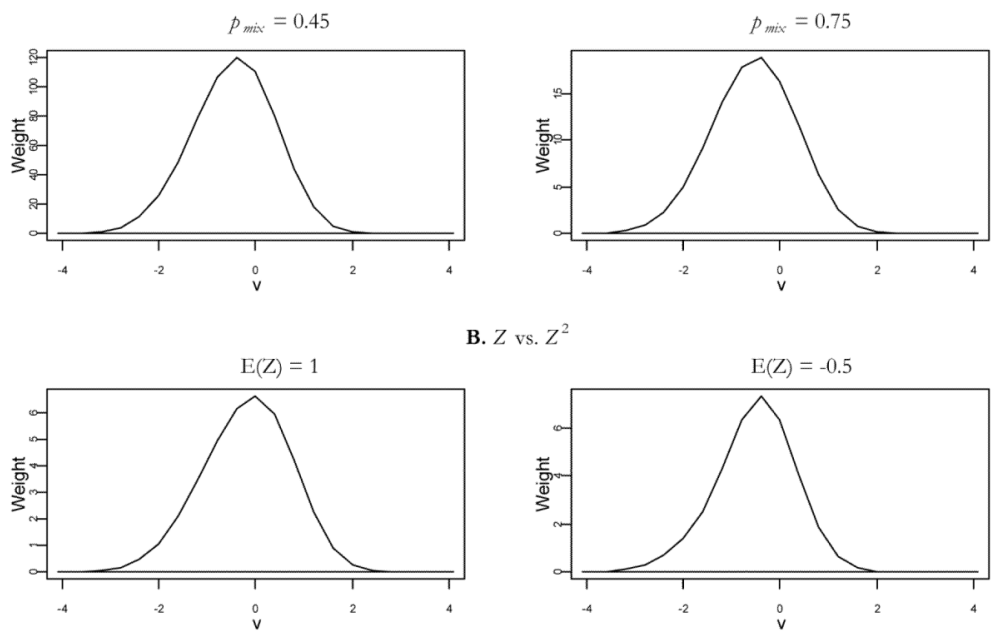

B. $Z$ vs. $Z^{2}$

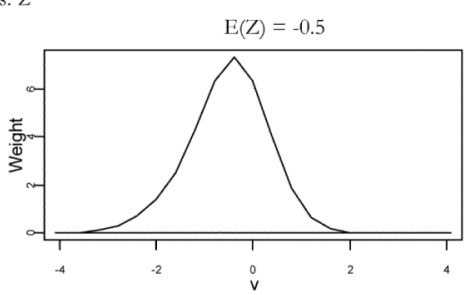

C. $\mathrm{P}(\mathrm{Z})$ above and below the median
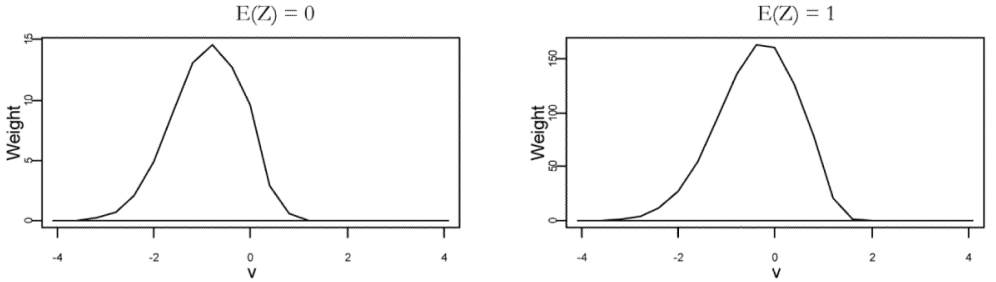

Figure 5.

IV variance weights $\left(h_{\Omega J_{1}, J_{2}}(\cdot)\right)$ as a function of $V=v$ for alternative choices of instruments. Note: Panel A plots the variance weights of the difference in the IV estimates constructed using either $Z_{1}$ or $Z_{2}$ as an instrument where $\left(Z_{1}, Z_{2}\right)$ is distributed as a multivariate mixture of normals, with $D=\mathbf{1}\left(\gamma_{1} Z_{1}+\gamma_{2} Z_{2}>V\right)$. To construct these results, we assume

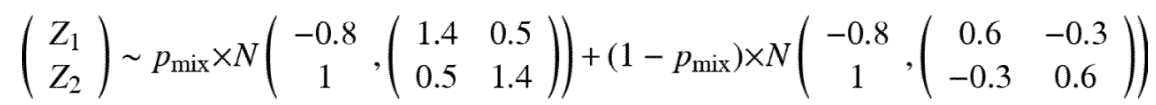

and the coefficients in the choice equation are $\gamma_{1}=0.2, \gamma_{2}=1$. In the left plot of Panel A we let $p_{\text {mix }}=0.45$ and in the right plot $p_{\text {mix }}=0.75$. Panel B plots the variance weights of the difference in the IV estimates constructed using either $Z$ or $Z^{2}$ as an instrument where $Z$ $N\left(\mu_{Z}, 1\right), \mu_{Z}=1$ or $\mu_{Z}=-0.5$, and $D=\mathbf{1}(Z>V)$. Panel $C$ plots the variance weights of the difference in the IV estimates constructed using either $P(Z)$ below the median or $P(Z)$ above the median as instruments. In Panel C, $Z \sim N\left(\mu_{Z}, 1\right), \mu_{Z}=0$ or $\mu_{Z}=1$, and $D=\mathbf{1}(Z>V)$. In all of the plots, we set $\sigma_{v}^{2}=1$. 


\section{Table 1}

Equivalence of Notation Between the Correlated Random Coefficient Model and the Generalized Roy Model. All parameters are defined conditional on $X_{i}$, which is left implicit

\begin{tabular}{lcc}
\hline & \multicolumn{1}{c}{$\begin{array}{c}\text { Generalized Roy } \\
\text { model }\end{array}$} & $\begin{array}{c}\text { Correlated random } \\
\text { coefficient model }\end{array}$ \\
\cline { 2 - 3 } Baseline outcome & $Y_{0, i}=\mu_{0}+U_{0, i}$ & $\alpha_{i}$ \\
Outcome in treated state & $Y_{1, i}=\mu_{1}+U_{1, i}$ & $\beta_{i}+\alpha_{i}$ \\
$\begin{array}{l}\text { Gain to treatment } \\
\text { (Individual causal effect) }\end{array}$ & $Y_{1, i}-Y_{0, i}=\mu_{1}-\mu_{0}+U_{1, i}-U_{0, i}$ & $\beta_{i}$ \\
Outcome & $Y_{i}=Y_{0, i}+D_{i}\left(Y_{1, i}-Y_{0, i}\right)$ \\
& $=\mu_{0, i}+\left(\mu_{1, i}-\mu_{0, i}+U_{1, i}-U_{0, i}\right) D_{i}+U_{0}$, & \\
\hline
\end{tabular}

